PROCEEDINGS OF THE

AMERICAN MATHEMATICAL SOCIETY

Volume 137, Number 11, November 2009, Pages 3717-3724

S 0002-9939(09)10031-X

Article electronically published on June 12, 2009

\title{
A NOTE ON A RESULT OF M. GROSSI
}

\author{
FLORIN CATRINA
}

(Communicated by Matthew J. Gursky)

\begin{abstract}
The purpose of this note is to present a fact complementary to a result in a recent paper of $\mathrm{M}$. Grossi. Making use of an energy balance identity, it is shown that the sufficient conditions for existence of solutions proved in Grossi's paper are also almost necessary.
\end{abstract}

\section{INTRODUCTION}

Let $N \geq 3$ and $\Omega \subset \mathbb{R}^{N}$ be a starshaped domain. It has been proved as early as 1965 by S. I. Pohozaev 18 that problems

$$
-\Delta u=u^{p} \text { in } \Omega, u=0 \text { on } \partial \Omega
$$

have no positive solutions for $p \geq \frac{N+2}{N-2}$. For $1<p<\frac{N+2}{N-2}$ the embedding $H_{0}^{1}(\Omega) \hookrightarrow L^{p+1}(\Omega)$ is compact and the existence of positive solutions can be proved by variational methods. For $p=\frac{N+2}{N-2}$ (i.e. $p+1=2^{*}=\frac{2 N}{N-2}$ the critical Sobolev exponent) the embedding is not compact anymore, and for $p>\frac{N+2}{N-2}$ the space $H_{0}^{1}(\Omega)$ is not a subspace of $L^{p+1}(\Omega)$ and the variational methods used to prove existence of solutions break down.

An interesting fact was observed by Brezis and Nirenberg in 3 relative to the problem

$$
-\Delta u=u^{2^{*}-1}+\lambda u, \quad u>0 \text { in } B, \quad u=0 \text { on } \partial B,
$$

where $B$ is the unit ball in $\mathbb{R}^{N}$ with $N \geq 3$. The authors proved that (2) admits a solution for positive values $\lambda$ contained in an interval whose endpoints depend on the dimension $N$ in a somewhat unexpected way. This paper gave rise to a flurry of work on problems with critical nonlinearities. It has been noticed that for some dimensions $N$ the branch of solutions which bifurcates from the trivial solution exists for all $\lambda$ between $\lambda_{1}$ (the first eigenvalue of $-\Delta$ with zero Dirichlet boundary conditions) and zero, while for other "critical" dimensions this branch is bounded away from $\lambda=0$ (see [2, 4], 6], [7, [10], [11, [12, [13], [16], [19], and the references therein).

Positive solutions for the more general critical exponent problem

$$
-\Delta u+a(x) u=u^{2^{*}-1} \quad \text { in } \Omega, \quad u=0 \quad \text { on } \partial \Omega,
$$

Received by the editors December 22, 2008.

2000 Mathematics Subject Classification. Primary 35J25, 35J70.

Key words and phrases. Green's function, positive solutions, supercritical exponent.

The author is grateful to the anonymous referee for useful comments and suggestions.

(C)2009 American Mathematical Society 
were discussed in [1, 3], 9], 17, 21], among many other works. As was made explicit by Druet 9], and earlier by Schoen 21] and Rey [20] (among others), Green's function of the operator $-\Delta u+a(x) u$ with zero Dirichlet boundary conditions on $\Omega$ plays an important role in the existence of solutions. For supercritical exponents, besides the early nonexistence results, there are few positive (existence) results (see e.g. [8] and the references therein).

In [15] the author looks at radial positive solutions for supercritical problems

$$
-\Delta u+a(|x|) u=u^{p}, \text { in } B, \quad u=0 \text { on } \partial B,
$$

where $a(r) \geq 0$ is a radial smooth function. By symmetry reduction, (3) becomes the ODE problem

$$
-u^{\prime \prime}-\frac{N-1}{r} u^{\prime}+a(r) u=u^{p}, \quad u>0 \text { in }(0,1), \quad u^{\prime}(0)=u(1)=0 .
$$

Let $H(r, s)$ be the regular part of Green's function for the operator on the left hand side, and with the boundary conditions, in (4). The central result of [15] states

Theorem 1. If there exists a nondegenerate critical point $\bar{r} \in(0,1)$ of the function $F(r)=\frac{H(r, r)}{r^{N-1}}$, then for large enough $p$ there exists at least one solution $u_{p}$ of (4).

We prove a somewhat complementary result. One way to state it would be in the form of the following:

Theorem 2. If the function $F_{p}(r)=\frac{H(r, r)}{r^{\frac{p-1}{p+3}(N-1)}}$ is monotonic, nonconstant, then problem (44) has no solution.

As $p \rightarrow \infty$ we have that $F_{p} \rightarrow F$ uniformly in any closed interval $\left[r_{0}, 1\right]$ with $0<r_{0}<1$. It is straightforward to see that if the hypotheses of Theorem 1 are met, then for sufficiently large $p$, the derivative $F_{p}^{\prime}(r)$ has to change sign, and therefore $F_{p}$ cannot be monotonic. In this sense, Theorems 1 and 2 complement each other.

The proof of Theorem 2 is based on an energy balance identity which has been developed in the paper of Catrina and Lavine [5]. The motivation in [5] was the refined Pohozaev identity used for the special case $N=3$ by Brezis and Nirenberg in 3 .

\section{Proof of Theorem 2}

Note that problem (4) can be written as

(5) $-\left(r^{N-1} u^{\prime}\right)^{\prime}+r^{N-1} a(r) u=r^{N-1} u^{p}, \quad u(r)>0$ in $(0,1), \quad u^{\prime}(0)=u(1)=0$.

Let $\xi$ and $\zeta$ be linearly independent solutions of the homogeneous equation

$$
-\left(r^{N-1} \xi^{\prime}\right)^{\prime}+r^{N-1} a(r) \xi=0 \quad \text { such that } \quad \xi^{\prime}(0)=\zeta(1)=0 .
$$

Observe that for $a \equiv 0$, a pair of such solutions is

$$
\xi_{0}(r) \equiv \frac{1}{N-2}, \quad \zeta_{0}(r)=r^{-(N-2)}-1
$$

According to our setting, we define the Wronskian of two functions to be

$$
W[u, \xi](r)=r^{N-1}\left(u(r) \xi^{\prime}(r)-u^{\prime}(r) \xi(r)\right),
$$

and note that

$$
W\left[\xi_{0}, \zeta_{0}\right](r) \equiv-1
$$


The proof of the following lemma will be sketched in the Appendix.

Lemma 1. For any bounded coefficient function $a \geq 0$, the problem (6) has a pair $\xi$, $\zeta$ of linearly independent solutions such that $\xi^{\prime}(0)=\zeta(1)=0$. Moreover, $W[\xi, \zeta](r) \equiv-1$ and the limits

$$
\lim _{r \rightarrow 0^{+}} r^{N-2} \zeta(r) \text { and } \lim _{r \rightarrow 0^{+}} r^{N-1} \zeta^{\prime}(r) \quad \text { exist and are finite. }
$$

We remark that the conclusions of the lemma above are valid for more general coefficient functions $a$, but for our purposes the present variant suffices. The next lemma states the energy balance identity that is at the center of the nonexistence result.

Lemma 2. With $\xi$ and $\zeta$ as in Lemma 1, any solution $u$ of (5) satisfies the identity

$$
\int_{0}^{1} r^{2(N-1) \frac{p+1}{p+3}} u^{p+1}\left(r^{\frac{4(N-1)}{p+3}} \xi \zeta\right)^{\prime} d r=0 .
$$

Proof. By multiplying the equation (6) by $-u$ and equation (50) by $\xi$ and adding, we obtain

Similarly we have

$$
\frac{d}{d r} W[u, \xi](r)=r^{N-1} u^{p} \xi
$$

$$
\frac{d}{d r} W[u, \zeta](r)=r^{N-1} u^{p} \zeta .
$$

By combining the two equalities above it follows that

$$
\frac{d}{d r}(W[u, \xi] W[u, \zeta])=r^{N-1} u^{p}(\xi W[u, \zeta]+\zeta W[u, \xi]) .
$$

Therefore

$$
\begin{aligned}
& \frac{d}{d r}(W[u, \xi] W[u, \zeta])=r^{2(N-1)} u^{p}\left(u(\xi \zeta)^{\prime}-2 u^{\prime} \xi \zeta\right) \\
& =r^{2(N-1)} u^{p+1}(\xi \zeta)^{\prime}-2 r^{2(N-1)} u^{p} u^{\prime} \xi \zeta \\
& =r^{2(N-1)} u^{p+1}(\xi \zeta)^{\prime}-\frac{2}{p+1} r^{2(N-1)}\left(u^{p+1}\right)^{\prime} \xi \zeta=\frac{d}{d r}\left(-\frac{2}{p+1} r^{2(N-1)} u^{p+1} \xi \zeta\right) \\
& \quad+r^{2(N-1)} u^{p+1}(\xi \zeta)^{\prime}+\frac{2}{p+1}\left(r^{2(N-1)} \xi \zeta\right)^{\prime} u^{p+1} .
\end{aligned}
$$

We now obtain

$$
\begin{aligned}
& \frac{d}{d r}\left(W[u, \xi] W[u, \zeta]+\frac{2}{p+1} r^{2(N-1)} u^{p+1} \xi \zeta\right) \\
& =\frac{p+3}{p+1} r^{2(N-1)} u^{p+1}(\xi \zeta)^{\prime}+\frac{4(N-1)}{p+1} r^{2 N-3} u^{p+1} \xi \zeta \\
& =\frac{p+3}{p+1} r^{2(N-1)} u^{p+1}\left((\xi \zeta)^{\prime}+\frac{4(N-1)}{p+3} r^{-1} \xi \zeta\right) \\
& =\frac{p+3}{p+1} r^{2(N-1) \frac{p+1}{p+3}} u^{p+1}\left(r^{\frac{4(N-1)}{p+3}} \xi \zeta\right)^{\prime}
\end{aligned}
$$

The lemma follows from the fact that the integral of the left hand side is zero. Indeed, from the boundary conditions and from Lemma 1 we have that

$$
\lim _{r \rightarrow 0^{+}} W[u, \xi](r) W[u, \zeta](r)=W[u, \xi](1) W[u, \zeta](1)=0
$$


and

$$
\lim _{r \rightarrow 0^{+}} r^{2(N-1)} u^{p+1} \xi \zeta=\left.r^{2(N-1)} u^{p+1} \xi \zeta\right|_{r=1}=0 .
$$

We are thus naturally led to define on the interval $(0,1]$ the function

$$
F_{p}(r)=r^{\frac{4(N-1)}{p+3}} \xi(r) \zeta(r)
$$

whose derivative appears in the energy balance identity. The following corollary of Lemma 2 is now immediate.

Corollary 1. If the dimension $N$, the coefficient function $a$, and the exponent $p$ in (4) are such that the function $F_{p}$ defined in (8) is monotonic, nonconstant, then the problem (4) has no solution.

Proof of Theorem 2. The only fact that needs to be checked is that the function $F_{p}$ defined in Theorem 2 is the same function as (8). Indeed, it is well known that Green's function of the operator

$$
-u^{\prime \prime}-\frac{N-1}{r} u^{\prime}+a(r) u, \quad \text { with boundary conditions } \quad u^{\prime}(0)=u(1)=0
$$

is given by

$$
G(r, s)=s^{N-1} \xi(\min (r, s)) \zeta(\max (r, s)), \quad \text { for } \quad r, s \in(0,1),
$$

where $\xi$ and $\zeta$ are functions satisfying (6) with the additional requirement that

$$
W[\xi, \zeta] \equiv-1 .
$$

We then observe that the regular part of Green's function as defined in [15],

$$
H(r, s)=G(r, s)-\Gamma(r, s), \text { where } \Gamma(r, s)=\left\{\begin{array}{l}
0 \text { if } r \leq s, \\
\frac{s^{N-1}}{N-2}\left(r^{2-N}-s^{2-N}\right) \text { if } r>s,
\end{array}\right.
$$

satisfies on the diagonal

$$
H(r, r)=G(r, r)=r^{N-1} \xi(r) \zeta(r) .
$$

Therefore

$$
F_{p}(r)=\frac{H(r, r)}{r^{\frac{p-1}{p+3}(N-1)}}=r^{\frac{4(N-1)}{p+3}} \xi(r) \zeta(r),
$$

i.e. the function that appears in (8).

It is instructive to analyze the case $a \equiv 0$ as $p>1$ increases from subcritical to supercritical. We have $F_{p}(r)=r^{\frac{4(N-1)}{p+3}} \xi_{0}(r) \zeta_{0}(r)$ with $\xi_{0}$ and $\zeta_{0}$ given by (77), i.e.

$$
F_{p}(r)=r^{\frac{4(N-1)}{p+3}} \frac{r^{-(N-2)}-1}{N-2} .
$$

The critical nonlinearity is given by $p=\frac{N+2}{N-2}$, and in this case $F_{p}(r)=\frac{1-r^{N-2}}{N-2}$, which is monotonic. Similarly, $F_{p}$ is monotonically decreasing for all $p$ supercritical, and we recover in this way Pohozaev's nonexistence result in the radial case. For $1<p<\frac{N+2}{N-2}$ we have

$$
\lim _{r \rightarrow 0^{+}} F_{p}(r)=F_{p}(1)=0
$$

and $F_{p}$ cannot be monotonic, which is consistent with the known fact that solutions of (4) with $a \equiv 0$ exist. In fact, in the subcritical case, solutions of (4) can be 
obtained by variational methods for a wide variety of coefficient functions $a$. In the case $a \geq 0$ and bounded, from Lemma 1 it follows again that

$$
\lim _{r \rightarrow 0^{+}} F_{p}(r)=F_{p}(1)=0
$$

and thus there is no obstruction.

As was mentioned in the introduction, if $F(r)=\frac{H(r, r)}{r^{N-1}}$ has a nondegenerate critical point $\bar{r}$, then so will have the function $F_{p}$ for $p$ sufficiently large. Under these circumstances, $F_{p}$ cannot be monotonic in $(0,1)$ and the energy balance identity is not an obstruction to the existence of solutions anymore. This is the case for subcritical nonlinearities.

\section{ApPEndix}

Even though the proof of Lemma 1 can be worked out directly on the equation (6) along the same lines as the proof below, for simpler notation we prefer to work with a transformed equation. Let

$$
s=\frac{1}{r^{N-2}}, \quad u(r)=v(s), \quad a(r)=b(s) .
$$

Then

Therefore

$$
\frac{d}{d r}=-(N-2) \frac{1}{r^{N-1}} \frac{d}{d s}=-(N-2) s^{\frac{N-1}{N-2}} \frac{d}{d s} .
$$

$$
u^{\prime}(r)=-(N-2) s^{\frac{N-1}{N-2}} v_{s}(s) \quad \text { and } \quad-\left(r^{N-1} u^{\prime}\right)^{\prime}=-(N-2)^{2} s^{\frac{N-1}{N-2}} v_{s s} .
$$

Hence, $u$ being a solution of the equation

$$
-\left(r^{N-1} u^{\prime}\right)^{\prime}+r^{N-1} a(r) u=0, \quad \text { for } \quad r \in(0,1)
$$

is the same as $v$ being a solution of the equation

$$
-(N-2)^{2} s^{\frac{N-1}{N-2}} v_{s s}+s^{-\frac{N-1}{N-2}} b(s) v=0, \quad \text { for } \quad s \in(1, \infty),
$$

or equivalently

$$
v_{s s}=\frac{c(s)}{s^{k}} v, \quad \text { where } \quad c(s)=\frac{b(s)}{(N-2)^{2}} \quad \text { and } \quad k=2 \frac{N-1}{N-2}>2 .
$$

Since we assumed that $a$, and therefore $c$, is a nonnegative bounded function, select $s_{0}>0$ such that

$$
\int_{s_{0}}^{\infty} \frac{c(t)}{t^{k-1}} d t \leq \frac{1}{2}
$$

For $s \geq s_{0}$, we define iteratively $v_{0}(s) \equiv 1$, and

$$
v_{n+1}(s)=1+\int_{s}^{\infty}\left(1-\frac{s}{t}\right) \frac{c(t)}{t^{k-1}} v_{n}(t) d t .
$$

By induction it follows that

$$
1 \leq v_{n}(s) \leq 2 \quad \text { for all } \quad n=0,1,2, \ldots, \quad \text { and all } \quad s \in\left[s_{0}, \infty\right)
$$

Also, note that for all $n \geq 1$ the functions $v_{n}$ are decreasing, and $\lim _{s \rightarrow \infty} v_{n}(s)=1$. On the other hand, for any $n \geq 1$ and for any $s \geq s_{0}$, we have that

$0 \leq v_{n+1}(s)-v_{n}(s) \leq \max _{\left[s_{0}, \infty\right)}\left(v_{n}-v_{n-1}\right) \int_{s_{0}}^{\infty} \frac{c(t)}{t^{k-1}} d t \leq \frac{1}{2} \max _{\left[s_{0}, \infty\right)}\left(v_{n}-v_{n-1}\right) \leq \frac{1}{2^{n+1}}$. 
Therefore, for any $n \geq m \geq 0$ it follows that for all $s \geq s_{0}$,

$$
0 \leq v_{n}(s)-v_{m}(s) \leq \frac{1}{2^{m+1}}+\frac{1}{2^{m+2}}+\cdots+\frac{1}{2^{n}} \leq \frac{1}{2^{m}} .
$$

It follows that $\left\{v_{n}\right\}_{n}$ converges uniformly on $\left[s_{0}, \infty\right)$ to a function $\varphi$ that satisfies

$$
\varphi(s)=1+\int_{s}^{\infty}\left(1-\frac{s}{t}\right) \frac{c(t)}{t^{k-1}} \varphi(t) d t,
$$

and therefore it solves (9). Since $1 \leq v_{n}(s) \leq 2$ for all $s \geq s_{0}$, it follows that $\varphi$ is bounded, positive, decreasing on $\left[s_{0}, \infty\right)$, and $\lim _{s \rightarrow \infty} \varphi(s)=1$. If $s_{0}>1$, we extend $\varphi$ backwards on $[1, \infty)$ so that it remains a solution of (9). Because it satisfies the equation, $\varphi$ remains decreasing on the whole interval $[1, \infty)$, and therefore $\varphi(1) \geq 1$.

We now define

$$
\psi(s)=\varphi(s) \int_{1}^{s} \frac{1}{\varphi^{2}(t)} d t .
$$

It is straightforward to check that $\psi$ is also a solution of (9), that $\psi(1)=0$, and that

We also have

$$
\varphi \psi_{s}-\varphi_{s} \psi \equiv 1 \quad \text { and } \quad \lim _{s \rightarrow \infty} \frac{1}{s} \psi(s)=1
$$

$$
\lim _{s \rightarrow \infty} \psi_{s}(s)=1
$$

Indeed,

$$
\psi_{s}(s)=\frac{1}{\varphi(s)}+\frac{\varphi_{s}(s)}{\varphi(s)} \psi(s)=\frac{1}{\varphi(s)}\left(1+s \varphi_{s}(s) \frac{\psi(s)}{s}\right),
$$

and (10) follows from $\lim _{s \rightarrow \infty} \varphi(s)=1, \lim _{s \rightarrow \infty} \frac{\psi(s)}{s}=1$, and by l'Hôpital's rule,

$$
\lim _{s \rightarrow \infty} s \varphi_{s}(s)=\lim _{s \rightarrow \infty} \frac{-\int_{s}^{\infty} \frac{c(t)}{t^{k}} \varphi(t) d t}{s^{-1}}=\lim _{s \rightarrow \infty}-\frac{c(s)}{s^{k-2}} \varphi(s)=0 .
$$

Let

$$
\xi(r)=\frac{1}{N-2} \varphi(s) \quad \text { and } \quad \zeta(r)=\psi(s)
$$

be solutions of (6). It follows that

$$
\varphi_{s}(s)=-r^{N-1} \xi^{\prime}(r) \quad \text { and } \quad \psi_{s}(s)=-\frac{1}{N-2} r^{N-1} \zeta^{\prime}(r) .
$$

We calculate

$$
\lim _{r \rightarrow 0^{+}} \xi^{\prime}(r)=-\lim _{s \rightarrow \infty} s^{\frac{N-1}{N-2}} \varphi_{s}(s)=\lim _{s \rightarrow \infty} s^{\frac{N-1}{N-2}} \int_{s}^{\infty} \frac{c(t)}{t^{k}} \varphi(t) d t,
$$

and by l'Hôpital's rule,

$$
\lim _{r \rightarrow 0^{+}} \xi^{\prime}(r)=\lim _{s \rightarrow \infty} \frac{N-2}{N-1} \frac{c(s)}{s^{k-\frac{2 N-3}{N-2}}} \varphi(s)=0
$$

because $k=\frac{2(N-1)}{N-2}>\frac{2 N-3}{N-2}$ and $c$ is bounded.

Hence $\xi$ and $\zeta$ satisfy the boundary conditions in (6).

We also have

$$
W[\xi, \zeta](r)=r^{N-1}\left(\xi \zeta^{\prime}-\xi^{\prime} \zeta\right)=-\left(\varphi \psi_{s}-\varphi_{s} \psi\right) \equiv-1
$$


and

$$
\lim _{r \rightarrow 0^{+}} r^{N-2} \zeta(r)=\lim _{s \rightarrow \infty} \frac{1}{s} \psi(s)=1
$$

The last fact left to justify is

$$
\lim _{r \rightarrow 0^{+}} r^{N-1} \zeta^{\prime}(r)=-(N-2) \lim _{s \rightarrow \infty} \psi_{s}(s)=-(N-2),
$$

because of (10).

\section{REFERENCES}

[1] H. Brezis, Elliptic equations with limiting Sobolev exponents - the impact of topology, Comm. Pure Appl. Math., 39 (1986) S17-S39. MR861481 (87k:58272)

[2] H. Brezis, L. Dupaigne and A. Tesei, On a semilinear elliptic equation with inverse-square potential, Selecta Math., 11 (2005) 1-7. MR2179651 (2006g:35052)

[3] H. Brezis and L. Nirenberg, Positive solutions of nonlinear elliptic equations involving critical Sobolev exponent, Comm. Pure Appl. Math., 36 (1983) 437-477. MR709644 (84h:35059)

[4] H. Brezis and L. A. Peletier, Elliptic equations with critical exponent on $S^{3}$ : New nonminimising solutions, C. R. Math. Acad. Sci. Paris, 339 (2004) 391-394. MR 2092750 (2005f:35090)

[5] F. Catrina and R. Lavine, Radial solutions for weighted semilinear equations, Commun. Contemp. Math., 4 (2002) 529-545. MR1918758 (2003e:35080)

[6] K.-S. Chou and D. Geng, On the critical dimension of a semilinear degenerate elliptic equation involving critical Sobolev-Hardy exponent, Nonlinear Anal. TMA, 12 (1996) 1965-1984. MR1386127 (97b:35079)

[7] Ph. Clement, D. G. de Figueiredo and E. Mitidieri, Quasilinear elliptic equations with critical exponents, Topol. Methods Nonlinear Anal., 7 (1996) 133-170. MR1422009 (97k:35072)

[8] J. Dávila, M. del Pino, M. Musso and J. Wei, Fast and slow decay solutions for supercritical elliptic problems in exterior domains, Calc. Var. Partial Differential Equations, 32 (2008), no. 4, 453-480. MR2402919 (2009b:35140)

[9] O. Druet, Elliptic equations with critical Sobolev exponents in dimension 3. Ann. Inst. H. Poincaré Anal. Non Linéaire, 19 (2002), no. 2, 125-142. MR1902741 (2003f:35104)

[10] H. Egnell, Semilinear elliptic equations involving critical Sobolev exponents, Arch. Rational Mech. Anal., 104 (1988) 27-56. MR956566 (90e:35068)

[11] H. Egnell, Existence and nonexistence results for $m$-Laplace equations involving critical Sobolev exponents, Arch. Rational Mech. Anal., 104 (1988) 57-77. MR956567 (90e:35069)

[12] P. L. Felmer and A. Quaas, Positive radial solutions to a 'semilinear' equation involving the Pucci's operator, J. Diff. Eqns., 199 (2004) 376-393. MR2047915 (2004m:34040)

[13] N. Ghoussoub and C. Yuan, Multiple solutions for quasi-linear PDEs involving the critical Sobolev and Hardy exponents, Trans. Amer. Math. Soc., 352 (2000) 5703-5743. MR1695021 (2001b:35109)

[14] B. Gidas, W. M. Ni and L. Nirenberg, Symmetry and related properties via the maximum principle, Comm. Math. Phys., 68 (1979) 209-243. MR544879 (80h:35043)

[15] M. Grossi, Radial solutions for the Brezis-Nirenberg problem involving large nonlinearities, J. Funct. Anal., 254 (2008), no. 12, 2995-3036. MR2418617 (2009c:35126)

[16] J. Jacobsen, Global bifurcation problems associated with $k$-Hessian operators, Topol. Methods Nonlinear Anal., 14 (1999) 81-130. MR.1758881 (2001f:35126)

[17] D. Passaseo, Some sufficient conditions for the existence of positive solutions to the equation $-\Delta u+a(x) u=u^{2^{*}-1}$ in bounded domains, Ann. Inst. H. Poincaré Anal. Non Linéaire, 13 (1996), no. 2, 185-227. MR1378466 (97i:35055)

[18] S. I. Pohozaev, On the eigenfunctions of the equation $\Delta u+\lambda f(u)=0$, (Russian) Dokl. Akad. Nauk SSSR, 165 (1965) 36-39. MR0192184 (33:411)

[19] P. Pucci and J. Serrin, Critical exponents and critical dimensions for polyharmonic operators, J. Math. Pures Appl., 69 (1990) 55-83. MR1054124 (91i:35065) 
[20] O. Rey, The role of the Green's function in a nonlinear elliptic equation involving the critical Sobolev exponent, J. Funct. Anal., 89 (1) (1990) 1-52. MR.1040954(91b:35012)

[21] R. Schoen, Conformal deformation of a Riemannian metric to constant scalar curvature, J. Differential Geom., 20 (1984) 479-495. MR788292 (86i:58137)

Department of Mathematics and Computer Science, St. John's University, Queens, NEW YORK 11439

E-mail address: catrinaf@stjohns.edu 